\title{
La Investigación Científica en la Historia y Cultura del Café en el Occidente de Honduras (La pequeña propiedad familiar)
}

\author{
PhD. Alfonso Carranza Ramos*1 \\ MsC. Jorge Humberto Orellana Peña*2
}

\section{Resumen}

Hablar del occidente de Honduras, es hablar del café, de la historia de su cultivo, que está asociado a la cultura que en su derredor se ha construido durante los últimos 150 años. La economía del café ha impulsado a la región occidental en términos de crecimiento y desarrollo al propiciar los procesos de reinversión más importantes del país, dando origen a la historia de una cultura que hoy constituye la más importante economía de los hondureños, junto con las remesas y la pequeña empresa, pilares en que descansa la paz relativa de esta región y de la nación.

\footnotetext{
* Investigadores Coordinación Regional de Investigación Científica- CRIC - CUROC. Santa Rosa de Copán, Honduras, Centro América, junio de 2013 Dirección: Villa Belén, Carretera hacia Gracias Lempira, Santa Rosa de Copán, Honduras. Tel. (504) 26623261,2662-1461, 2662-3223. jorellana4000@yahoo.es, alfonsocarranzar@yahoo.es

1 Alfonso Carranza Ramos: Ingeniero Agrónomo de la Universidad Nacional Autónoma de Honduras, con estudios de Maestría en Planeación y Desarrollo por CEDEFT-México-UNAH. Con estudios de Doctorado en Educación por la Universidad de La Salle, Costa Rica. Investigador de Proyectos de Desarrollo, consultor, caficultor, historiador y profesor del Centro Regional Universitario de Occidente. Ha publicado varias obras y ha dedicado los últimos veinticinco años al estudio de la realidad de las familias campesinas en el occidente de Honduras.

2 Jorge Humberto Orellana: Licenciado en Comercio Internacional por la Universidad Nacional Autónoma de Honduras, con estudios de maestría en Desarrollo y Cooperación Internacional por la Universidad del País Vasco en Bilbao España, investigador, historiador, conferencista y profesor titular del Centro Regional Universitario de Occidente, UNAHCUROC. Ha laborado para prestigiosas Organizaciones no Gubernamentales y ha dedicado los últimos años al estudio del desarrollo, historia y ambiente, especialmente en la región occidental de Honduras. Autor de varias publicaciones acerca de temas ambientales, historia y cultura. Sus áreas de especialización en investigación son: Desarrollo, Cultura y Ambiente, Historia, Cooperación Internacional, Construcción de Ciudadanía, Empresa Familiar.
} 
Este trabajo, presenta el desarrollo histórico y cultural de uno de los rubros de producción en los cuales se sustenta la economía regional y nacional. El café constituye para 35,000 familias aproximadamente del occidente de Honduras, su forma de vida, con su propia dinámica de reinversión en sus ciclos productivos, con experiencias de diversificación interesantes de estudiar y comprender. Sus plantaciones han sido factor decisivo para aproximarse a mejores condiciones de bienestar, a una mejor distribución del ingreso, acercar oportunidades y posibilidades, a ser parte de ambientes naturales.

Las universidades a través de los procesos de vinculación e investigación científica, encontrarán en la historia y cultura del café, interesantes campos de estudio; cadenas de valor, experiencias de éxito, conocimientos locales que permitirán la construcción de aprendizajes y saberes desde dentro, dinámicos y complejos, elementos que proporcionarán nuevas probabilidades para construir nuevos caminos, donde la población sea parte y especialmente, con visiones incluyentes para nuestra sociedad.

Palabras Claves: <Historia $><$ Café $><$ Cultura $><$ Investigación $><$ Familias $><$ Productores $><$ Universidades $><$ Sistematización $>$

\begin{abstract}
Abstrac
Speaking of western Honduras, is to speak about coffee, the history of cultivation, which is associated with the culture around him has been built over the last 150 years. The coffee economy has thrust the western region in terms of growth and development processes to encourage reinvestment in the country, giving rise to the story of a culture that today is the most important Honduran economy, together with the family remittances and small business, pillars on which rests the relative peace of this region and the nation.

This paper, presents the historical and cultural development of one of the production areas on which is based the regional and national economy. Coffee is for approximately 35,000 families in western Honduras, their way of life, with its own dynamic of reinvestment in their production cycles, with diversification experiences interesting to study and understand. Its plantations have been crucial for approaching best welfare conditions, better income distribution, brings opportunities and possibilities, to be part of natural environments.
\end{abstract}


The universities through linking processes and scientific research, will find in the history and culture of coffee, interesting fields of study; value chains, success stories, local knowledge that will enable the construction of learning and knowledge from within, dynamic and complex, elements provide new probabilities to build new ways, where the population is part and especially, with inclusive visions for our society.

Key words: $<$ History $><$ Coffee $><$ Culture $><$ Research $><$ Families $><$ Farmers $><$ Universities $><$ Systematization $>$

\section{Introducción}

El cultivo del café constituye para la región del occidente de Honduras su alternativa; siendo que es el resultado de un proceso que comenzó hace más de 150 años, tiempo en el cuál se ha recorrido un largo y difícil camino, llegando hoy, a ser la región más importante del país en ese rubro y convirtiéndose en una zona productora de exquisitos cafés. A pesar de la pirámide de desigualdad, dura y evidente, principalmente en los procesos de acopio, control, intermediación, comercialización y exportación, las pequeñas fincas productoras de café se han constituido en la base de la economía regional y en la ventana de distribución de recursos entre las familias propietarias de pequeñas fincas y el sector de cortadores. Son dos economías en la cadena de un solo rubro, desde su producción hasta su comercialización; una economía social de la finca a su interior y una economía capitalista impositiva de la finca hacia el exterior.

En este artículo, buscamos acercarnos a la comprensión de la historia y cultura del café en el occidente de Honduras, a la pequeña propiedad familiar; para ello, se plantea una visión desde la trascendencia del cultivo; algunos criterios sobre los conceptos de cultura y las condiciones biofísicas del occidente, con énfasis en las relaciones agroecológicas y sus configuraciones humanas histórica culturales. Se presenta además, una mirada histórica desde la llegada del café a Honduras y a la región occidental; sus etapas de desarrollo, fomento y su cadena de valor; destacando la importancia de la pequeña propiedad familiar, como base de la socio economía del país. Finalmente se analiza la oportunidad que tienen los sectores académicos, principalmente las universidades, de acercarse a los 
pequeños productores de café con sus experiencias de éxito: esto es acompañar en el proceso, desde la academia, es profundizar con una visión incluyente en el estudio, entendimiento y comprensión de realidades locales como las que vive la región occidental de Honduras.

\section{Cultura y trascendencia del cultivo del café en el occidente de Honduras}

La cultura es una cualidad de los seres vivos, se manifiesta de maneras diferentes y con distintas intensidades. La cultura encierra esos maravillosos mundos de la trilogía; sociedad, especie e individuo, que Edgar Morín plantea: Las culturas son interacciones, intercambios, procesos de construcción colectiva. Llamamos cultura -en los humanos- a las costumbres que se van desarrollando, por un individuo o por una sociedad, en un tiempo dado y en un espacio o circunstancias físicas determinadas. Las culturas son construcciones de carácter social en la cual los seres pertenecientes de una u otra forma aportan al grupo y son influenciados, a su vez, por aquel. Cultura es conocimiento acerca de algo, o mejor dicho, es conocer algo. Es la colectividad que se manifiesta de manera individual; pero la cultura es por así decirlo, una serie de procesos de creación, de mentes colectivas.

La cultura implica costumbres, hábitos, formas de pensar, de hacer y sentir, de construir e interpretar los fenómenos. Pero también implica crear, construir e inventar, como también formas nuevas de hacer las cosas viejas; creando las técnicas; por ejemplo en los cultivos, las variedades, los gustos y preferencias proceden de estos. Clyde Kluckhohn planteóen su obra Mirror for manque la cultura es el "modo total de vida de un pueblo, el legado social que el individuo adquiere de su grupo, una manera de sentir y creer, un depósito de saber almacenado, una abstracción, un mecanismo de regulación" (1973, p. 20).La cultura puede entenderse como un concepto semiótico; símbolos que expresan significados sociales que hacen conexiones, sentidos e imágenes. Geertz dice: "La cultura consiste en estructuras de significación socialmente establecidas" (Clifford, 1973, p. 26). 
La cultura de las sociedades agrarias está asociada con los ambientes, que a su vez influencian al agro del cual dependen, forman una serie de círculos, de redes o cadenas que articulan diversos saberes, percepciones y sentires; esa cultura conserva, modifica y cambia a la vez. En la lógica de sobrevivencia ${ }^{3}$ de las familias pobres del occidente de Honduras, han logrado sobreponerse a las arbitrariedades sin límites, a las desventajas históricas de exclusión, porque con tan escasos recursos disponibles, con lo poco que han tenido, hacen el milagro de la vida (una pequeña finca menor de 5 manzanas mantiene una familia de cinco miembros, con una explotación de más de un $85 \%$ de su superficie). Hay una complementariedad entre la cultura que se va articulando con los entornos, esto hace que el conocimiento de los mismos ambientes, sean parte de esas culturas locales, de allí que la gente que vive en el campo, sabe tanto de las características y detalles de su entorno (Carranza, 2012, p. 25).

Clifford (1973) señala "que no existe una naturaleza humana, independiente de la cultura" (Op. cit., p. 55). Esta tiene mayor relevancia cuando de esos saberes y conocimientos se depende para vivir, para cubrir las necesidades básicas. Hablamos entonces, de culturas más específicas como "la Cultura del Maíz, la Cultura de la Carencia, la Cultura del Café". Por ejemplo, Rivas (2003) en su tesis doctoral de la Universidad de Gotinga, Alemania, encontró en el Valle del Cauca, Colombia, que el conocimiento de los campesinos sobre la fertilidad de sus suelos era muy avanzado, color, olor, textura, materia orgánica (p. 1); mientras científicos de la Universidad de Berkeley en California, argumentan que los campesinos de origen Tzeltal en Chiapas, saben tanto de insectos como un buen entomólogo (Toledo, 1994, p. 74). Si a eso añadimos la distancia en el tiempo y el conocimiento "heredado" entre generaciones, hablamos entonces de variables culturales cuyas maneras de ver el mundo tienden a ser más perdurables. La historia y cultura de nuestros pueblos se escribe con las manos, con la piel viva y con los pies descalzos; nace de raíces hundidas, junto al maíz, los frijoles y el café, nace con el barro; es de tierra, de olvido y también de silencio. La historia junto con la cultura y las

${ }^{3}$ Usamos la palabra "sobrevivencia" en sustitución de "supervivencia". Considerando que la palabra "sobrevivencia" ha tenido más significado histórico en la región occidental de Honduras. 
características de la región del occidente de Honduras, constituyen los elementos principales de la sociedad que conocemos.

\section{Condiciones biofísicas de la región occidental de Honduras}

La región del occidente es geológicamente la más antigua; se origina junto con la región del sur de México y norte-centro de Guatemala, después de sucesivas y grandes inestabilidades (Flores y Palacios, 2009, p. 21). Así mismo, occidente es la más agreste del país, lo que permite condicionar una serie de microclimas, con alta biodiversidad, alturas que van entre los $450 \mathrm{msnm}$, superando los 2850 msnm y con variadas condiciones de temperatura, humedad y precipitación pluvial. Las fincas están ubicadas en las zonas más altas, los cafés de mejor calidad se producen sobre los 1,100 msnm. La región posee cinco macizos montañosos: Celaque, Güisayote, Puca-Acangual, Erapuca y Camapara, sus propias características agroclimáticas junto con el manejo, determinan las condiciones de la producción del café. "La región del occidente de Honduras está formada por las cadenas montañosas del Merendón, que nacen en la República de Guatemala, cruzando nuestro país de occidente a nor-oriente por los departamentos de Copán, Lempira, Santa Bárbara, ramificándose y formando las cuencas de los ríos Chamelecón y Motagua” (Castellanos, 1980, p. 15).

Apenas un $2.5 \%$ de su territorio es plano, especialmente en los valles de Sensenti y Cucuyagua al norte del departamento de Ocotepeque y al Sur del departamento de Copán. Está comprendida en la macro región denominada Mesoamérica, una de las más importantes del mundo en términos de biodiversidad, extendiéndose desde el sur de México hasta Centro América, y según los científicos rusos Vavilov y Bukasor, quienes a principios del siglo pasado hicieron estudios sobre las cinco grandes regiones y centros geográficos del origen de las plantas en el planeta, esta región se considera junto con la Amazonia, las que poseen la más rica biodiversidad de la tierra. Las posibilidades de ambientes en el occidente de Honduras permite que la vida se desarrolle en sus más diversas condiciones; para la agricultura del café, está entre lo mejor por la calidad de sus suelos, temperaturas, precipitación, radiación solar, alturas sobre el nivel del mar, microbiología, textura, entre otras. 


\section{Las antigual poblaciones de la región}

La configuración de las poblaciones del occidente de Honduras está relacionada con su lejana y cercana historia. Las antiguas civilizaciones que habitaron estas tierras se remontan a la memoria de los siglos, a más de cinco mil años de su presencia. Entre las más importantes culturas mencionaremos a los Lencas y Maya-Chortí, como parte de la familia Maya-Mayense, con sus variantes MayaChortí, Maya-Chol, Maya-Chontales; que hasta nuestros días habitan parte del departamento de Ocotepeque, Copán y la zona del oriente de Guatemala. El Centro Ceremonial de Copán, que floreció entre los siglos V y VII de nuestra era, se considera que fue parte de la antigua civilización Maya. Cultivaron maíz, frijoles y calabazas; sus sistemas de asocio, son hasta nuestros días la base de los sistemas tradicionales de producción. Su arquitectura y sus conocimientos son todavía -trece siglos después- asombrosos.

Los Lencas por su parte, menos visibles, fue la etnia más numerosa en el siglo XV y XVI, superando las 350,000 personas. Varios autores como Linda Newson y José María Tojeira, han señalado las diferentes teorías sobre sus orígenes; mientras Barón Castro, sostiene que fueron los remanentes directos del antiguo imperio Maya (Rivas, 2000 , p. 57). Se agruparon en cuatro grupos lingüísticos: Cares, Potones, Cerquines, Taulepas. Su agricultura representó, como en los Mayas, la base de su vida, sus sistemas de rotación y relevo de cultivos, sus sistemas de asocio (maíz, frijol milpero, ayote, otras plantas), para diferentes ambientes, zonas frías y cálidas, el uso de la mano de obra, las tierras en barbecho (como sistema agroforestal), las diferentes variedades de maíz, maíces tempranos y tardíos, maíces de porte alto más productivos y de porte bajo menos productivos, maíces para zonas frías y cálidas; sus sistemas de almacenamiento en trojas curadas con calor (fuego), sus sistemas de selección de semillas fueron elementos trascendentales de su sistema agrícola.

Marvin Barahona en su obra "Evolución histórica de la identidad nacional" plantea que la agricultura campesina de subsistencia, tiene su base en esos sistemas. Carranza por su parte, escribe que la versatilidad de esta agricultura ha permitido que las mayorías de 
familias campesinas pobres encuentren sus alimentos por esta vía, aunque no se estudien los antiguos sistemas tradicionales de producción (Carranza, 2004, pp. 65 - 67). Cuando llegaron los españoles en el siglo XVI, el grupo indígena de los Lencas eran los más numerosos. Con el proceso del mestizaje, la dominación militar, económica e ideológica, dio como resultado una población indígena disminuida, vencida y sometida, en cuyas tierras se establecieron aquellos conquistadores recién llegados. De hecho en 1536 se reparte la tierra de la ciudad que llevaría por nombre Gracias a Dios, (hoy ciudad de Gracias, Lempira), don Juan Bautista y unos señores de apellido “De la Fuente”, don Perafán de Rivera, fueron los encomenderos en esas tierras (Pérez, A, Pérez, H., y Pérez, T., 1936, p. 135-142).

La disponibilidad de la mano de obra fue el factor más importante en el proceso de la consolidación de las encomiendas. La fuerza laboral indígena constituyó la verdadera riqueza de la base de la economía colonial; desde el inicio de la colonia la mano de obra indígena estuvo en todo, era la fuerza que movía al mundo; en la agricultura, minas, en las casas, en la ganadería, sembradíos de caña en las Antillas (hasta allá llegó la mano de obra del occidente de Honduras, especialmente de Lempira e Intibucá), en los ejércitos, en las construcciones importantes (en 1759 se comenzó a construir el Fuerte de San Fernando de Omoa, con mano de obra de esta región). Las estructuras del poder colonial en el usufructo de la tierra fue heredada por el régimen que le sucedió, las estructuras del poder sobre la tierra se intensificó durante el régimen de Marco Aurelio Soto en Abril de 1877, cuando publicó la Ley de Agricultura, permitiendo adquirir tierras a quien tuviera la capacidad de comprarlas (Molina, 1985, p. 46). Todos estos factores han sido básicos para determinar las condiciones que marcarían las sociedades y economías de nuestra región occidental y del país en general.

\section{La llegada del café a Honduras}

El café se extendió por el mundo a raíz de que los holandeses y los franceses lo llevaron a sus respectivos países desde Etiopía y Abisinia (al noreste de África), tiempo después el café fue introducido en América entre los años de 1714 y 1728 (Montes, 1928, p. 26). El 
café llegó a las colonias de Surinam, Martinica, Santo Thomás, La Española (República Dominicana), Haití y Cuba, a escala grande relativamente (Alvarado, 1936, p. 673). A Costa Rica llegó en 1790 y a Honduras en 1799, a través del puerto de Trujillo, en una goleta llamada Reyna Luisa, el 12 de septiembre de ese año. De esa forma, desde la isla de Cuba, trajeron a Honduras los primeros "seis quintales de café" (Carranza, Op. cit., p. 57).

Posteriormente, los registros del puerto de Trujillo (principal puerto de Honduras en esa época), documentan que en 1800 llegó café en grano, café molido en barriles desde esa isla (Rubio, 1975, p. 86). Junto con el grano llegaron también ideas, tecnologías de su siembra, cosecha y usos. A finales del período colonial en las ciudades de Trujillo, Comayagua y Gracias a Dios, funcionó una especie de jardines botánicos, creados con la finalidad de reproducir y mantener vivas las plantas que desde Europa y de otros lugares del mundo se trajeron a Honduras. El Intendente don Ramón Anguiano, en su visita a Honduras en 1801, acerca del café de Honduras expresó en su informe "el café es tan de excelente calidad, como el de Moka" (Vallejo, 1893, p. 120).Criterio de muy alta consideración porque el puerto de Moka, era el lugar de Yemen (en la península Arábiga) cerca de Abisinia (África) por donde, en aquel entonces, se exportaba el mejor café del mundo.

\section{La llegada del café al occidente de Honduras y el desarrollo de su cultivo}

Los primeros datos que se tienen del cultivo del café en el occidente de Honduras son de 1850, cuando es introducido a la comunidad de Llama, Santa Bárbara, en un relato publicado en 1940 por don Rubén Ángel Rosa, en su obra: Monografía del Municipio de Llama, nos dice: "En Honduras, se comenzó a cultivar en el Departamento de Santa Bárbara, en el Municipio de Llama por Don Leandro Rosa y Don Cenón Mencía, quienes trajeron de la capital de Guatemala, en noviembre de 1850 , obsequiados por el Dr. Luna, algunos arbolitos de café". Don Leandro Rosa, "militó bajo las órdenes del general Cabañas" (Rosa, 1940, p. 13).

Otras apreciaciones consideran que el café llegó a esta región entre 
los años de 1835 y 1850 . Se habla también de don Gregorio Rivera, quien habría llevado café al Municipio de Colinas en esos años. En 1850 el cultivo del café ya estaba en plena expansión en los países vecinos de El Salvador y Guatemala, los que habían conocido a su vez, el auge del mismo en los cultivos de Costa Rica. Se considera también que trajeron café de El Salvador al occidente de Honduras, dados los nexos familiares, comerciales y culturales entre los pueblos; Gualcinse, San Marcos de Ocotepeque, Corquín Copán, fueron parte de esos acontecimientos.

El desarrollo del cultivo del café en el occidente de Honduras se puede estructurar en tres grandes etapas: a) Como planta medicinal de jardín, b) El café como planta de solar, c) Como cultivo hacia la producción y comercialización. Cuando el café llegó a Honduras fue considerado una planta maravillosa y medicinal. Era de uso común intercambiarse plantas y semillas de interés, especialmente entre gente más informada. La etapa de solar es cuando el café, poco a poco, es conocido y se constituye en parte de las plantas del solar cerca de la casa o en los alrededores de la vivienda; es allí donde la población conoce de su cultivo; observa sus requerimientos, sus ciclos, desarrolla sus criterios, lo estudia, contextualiza y lo consume, fue una etapa de posicionamiento cultural. Asimismo, junto a esto se desarrolló también una mayor demanda del grano, que constituyó también un elemento clave en la cultura de la región en cuanto al consumo del café. Posteriormente la etapa del café como cultivo de exportación tomó mayor visibilidad con las reformas del gobierno de Marco Aurelio Soto y Ramón Rosa.

Así, en el proceso de integración del café en la vida de la gente pasando a ser parte de su diario vivir, es significativo el sentido que culturalmente se le asigna: El café es alimento, es sabor, es medicina, ingreso, valor de cambio -se usó por mucho tiempo como papel moneda-, es trabajo y también es época. La iniciativa individual, la curiosidad, las políticas municipales y posteriormente las políticas del Estado, contribuyeron a la difusión del café en esta región del occidente de Honduras. La Municipalidad de Corquín en 1873, hace constar que en ese municipio, en esa época, hay establecidas más de 450 manzanas de café, al que consideran su patrimonio, con un promedio de 3 a 4 manzanas por finca; es el documento más 
completo que sobre el cultivo del café se haya formulado desde una instancia municipal.

Mientras en Trinidad Santa Bárbara, el doctor Alejandro Salomón Sagastume Fajardo dice: "Desde antes de 1850, se cultivaba café, que no pasaba de ser un producto para el consumo local", luego añade: "el gobierno local concedió muchas tierras ejidales a familias como los Paredes, Pineda, Rivera, Rápalo y otras, dedicadas al cultivo del café" (Stanley, 1994, p. 15). La difusión del café era una novedad, en 1845 en El Salvador se publicó un libro acerca del café donde se encumbraban las ventajas del cultivo y se miraba como ejemplo a Costa Rica. En el año de 1849 el presidente Coronado Chávez publicó un decreto para el fomento del cultivo del café. En 1860 el general José María Medina promovió el cultivo de esta planta en la zona del valle de Sensenti y San Marcos de Ocotepeque. En 1877 con la emisión de la "Ley de Agricultura" del gobierno de Marco Aurelio Soto y Ramón Rosa, intentaron dinamizar este cultivo desde una perspectiva de la economía liberal, hacia el mercado internacional. En 1862 el presidente Gerardo Barrios de El Salvador había promovido una ley para el fomento de la caficultura. Durante esa época, el general Justo Rufino Barrios, presidente de Guatemala y caficultor reconocido, había tenido entre sus más importantes ministros, a los hondureños Marco Aurelio Soto y Ramón Rosa, en quienes ejerció una gran influencia, principalmente bajo la concepción liberal, que el cultivo del café podría desarrollar la economía nacional al construir políticas que facilitaran su expansión, especialmente para los inversionistas extranjeros. Esto podría insertar en el mercado mundial a nuestro país; ese fue el objetivo de la ley de agricultura anteriormente comentada.

\section{Las fincas familiares del occidente de Honduras}

Hubo tres factores básicos del por qué las fincas hasta hoy continúan siendo pequeñas en el occidente del país: a) Las condiciones de heterogeneidad de los suelos, sus condiciones de ladera y su origen, b) Los procesos políticos y socioeconómicos que definieron la estructura agraria; es el Estado el mayor dueño y la dinámica política que esto ha generado (de hecho, los más favorecidos fueron siempre los de mayor poder económico y poder político; las grandes 
propiedades en el occidente tienen su origen en la "herencia política”), c) El movimiento económico que el café genera, a partir de ser considerado un rubro de ingresos, donde poco a poco se reinvierte, a pesar de los escasos márgenes de rentabilidad, pero en esa posibilidad se añade "una parcela más", y la familia crece y "hay que dejarle a todos", en una dinámica de la socio economía regional, propia del paradigma de la subsistencia (Carranza, Op. cit., p. 90).

Don Felipe Reyes en 1927 decía proféticamente: “La pequeña propiedad incrementará positivamente la economía nacional. Ella es la base de los pueblos y promueve su arraigo" (Reyes, 1927, p. 3). Precisamente, la economía de Honduras depende básicamente de la pequeña empresa cafetalera de carácter familiar, la micropequeña-mediana empresa, y las remesas enviadas desde el exterior. Por ello, las fincas de café del occidente tienen un carácter de empresa familiar (informal); con tamaños de área promedio de 3 manzanas y con más de 35,000 familias dedicadas a este cultivo, quienes logran una producción que en la cosecha 2011-2012 alcanzó el 38\% del total de la producción nacional (Carranza, Op. cit., pp. 18-19). Las familias dedicadas al café, son las que más reinvierten en la región; su dinámica incluye diferentes campos: ganadería, especies pequeñas, agricultura, comercio y transformación de materias primas. Así mismo, es el sector que más contribuye a la educación, genera trabajo y junto a los otros mencionados, hace de la misma el tejido socioeconómico más importante de sus procesos de desarrollo. Es esa dinámica la que permite que el país tenga todavía en estas regiones una esperanza de vida, de sueños y aspiraciones.

Fundamentalmente, toda economía en nuestro medio tiene la forma de una pirámide; a mayor altura, menor base, lo que implica mayor desigualdad (triángulo isósceles). A mayor desigualdad, mayor exclusión. En el caso del café hasta un 25\% del costo bruto de producción se va hacia los sectores más pobres, los propios cortadores, distribuyendo de esta forma el ingreso que el café genera. En la comercialización el proceso es diferente, fuera de la finca, intermediarios en sus distintos niveles, son quienes disfrutan de una mejor remuneración por su intervención en la cadena de 


\section{La pequeña propiedad familiar del café y el aporte de la investigación científica desde las universidades}

La calidad del café en el occidente de Honduras, es un hecho de construcción social participativo en interacción con lo genético ambiental. La calidad se afina con la experiencia, es más que la sumatoria de una serie de procesos coordinados y coherentes; es creatividad, cultura y espíritu. Cada productor conoce tal calidad por su proceso productivo, por la textura, color del grano, su olor, forma y tamaño, que se asocia a la variedad y altura sobre el nivel del mar. Las familias productoras de café por décadas han ido perfeccionando sus cosechas, su valor agregado; así, desde aquellas familias precursoras como los Enamorado, Medina, Paz y Castellanos en el departamento de Santa Bárbara; los Rosa Bautista en Gracias Lempira, la familia Rodezno Pineda Espinoza en San Marcos de Ocotepeque, productores copanecos como los Welchez en Copán Ruinas, los Urquía en San Juan de Opoa, la familia Romero y los Deras Reyes en el municipio de Corquín Copan, son quienes han hecho historia en la cultura del café del occidente del país.

En este sentido, las Universidades-especialmente las universidades públicas - tienen ante la patria el compromiso ineludible de profundizar en el conocimiento de las regiones, pero solo se puede profundizar, al estudiar, conocer y comprender; esto no se puede lograr sin investigar, sin auscultar los intereses temáticos de una región, sin estudiar los contextos, su historia y su cultura, su gente con sus técnicas y procesos. En la UNAH, por mandato legal se debe hacer, de acuerdo con la ley orgánica; pero además, lo que realmente trasciende es la necesidad de conocer más, de conocer mejor, para que los aportes desde la Universidad a través de la educación sean más efectivos para el contexto regional y nacional. Por lo tanto, la investigación en las universidades debe contribuir a la comprensión de esas realidades regionales, tan complejas y dinámicas a la vez, y de ese estudio y conocimiento, pueden construirse propuestas de nuevas interpretaciones y procesos de cambio, hacia mejores condiciones en las que podrían vivir las poblaciones hasta ahora desposeídas. 
Conscientes de tal necesidad, el Centro Regional Universitario de Occidente UNAH-CUROC, a través de un equipo de investigación, en los últimos meses acompañó un proceso de conocimiento y aprendizaje en una pequeña finca de la familia Pacheco Estévez en la comunidad de Jimilile Corquín, Copán. El trabajo consistió en sistematizar la experiencia de esta propiedad familiar dedicada a varios rubros de producción. En la extensión de la finca, principalmente se produce café, pero sumado a ello, cultivan tilapia, miel y árboles frutales que complementan la dieta familiar. "El conocimiento adquirido a través de la experiencia sistematizada en "Finca las Lichas", refuerza nuestro compromiso como academia de indagar y comprender los problemas que viven las familias del occidente del país, con premisas tendientes a ofrecer alternativas 0 propuestas para una mejor intervención" (Orellana, Landaverry y Miranda, 2013, p. 13).

El trabajo de sistematización con un enfoque de investigación y componentes de vinculación Universidad - Sociedad, describe de forma analítica los factores que han permitido el desarrollo de una finca convencional hacia una que integra diversas actividades productivas; relaciones familiares, gestión y fortalecimiento empresarial y un cuidado del entorno. La familia Pacheco Estévez, por varias décadas ha fomentado una cultura de solidaridad, de unión familiar, de respeto por las personas mayores, con enfoque hacia la productividad y cuidado de la Tierra. La producción del café ahora pasa por un proceso de valor agregado, han patentado la marca "Café Jimilile", la cual compite de forma exitosa en el mercado por su alta calidad. Don Justino, patriarca de los Pacheco, ha sabido inculcar en su núcleo familiar una serie de valores que han posibilitado una cultura de emprendedores. El amor y el trabajo en equipo ha sido su filosofía, la cual es compartida con todos sus hijos, esto ha permitido una mejor coherencia familiar y una mayor asimilación en la transmisión de conocimientos. Con la llegada y el apoyo de algunas organizaciones de desarrollo como ODECO, IHCAFE, PLANDERO entro otras, se crearon y fortalecieron una serie de capacidades técnicas y financieras, esto permitió el surgimiento de una visión de diversificación y mejoramiento permanente de la calidad productiva, alcanzando procesos interesantes de certificación. La finca ha logrado obtener sellos de calidad internacional como: 
“Orgánico, Comercio Justo y Rainforest”.

Como equipo de investigación del CUROC, la experiencia ha permitido valorar el verdadero papel que debe desempeñar la academia en los temas de "Vinculación Universidad - Sociedad e Investigación Científica". Quizá, la familia Pacheco Estévez no sea la más "pudiente" en la región, pero nos ha interesado su legado, debido a que una familia no se define por un apellido en común, sino la común - unión que se erige de los valores, se expande con la buena práctica y encumbra con la supremacía del amor y el respeto. Acercarnos a estas experiencias, estados o realidades que vive la población del occidente del país, especialmente en la pequeña propiedad familiar, posibilita a estudiantes y docentes de la academia, la búsqueda de preguntas o respuestas a los múltiples problemas estructurales que enfrentan nuestras comunidades de la región occidental de Honduras.

Este debería ser el aporte de la investigación científica desde las universidades; conocer y conocernos, ciencia con conciencia, búsqueda, encuentros y reencuentros. No se trata solamente de reproducir, es aprender y crecer; aprender por ejemplo de las pequeñas familias productoras de café con su "mundo de saberes locales", quienes fueron construyendo conforme se aprendía "a prueba y error", pero que en el tiempo han logrado aprendizajes interesantes, paradójicamente en su mayoría, desconocidos en la academia. Por ende, se trata de la búsqueda y la construcción de nuevos conocimientos desde dentro, desde lo local, con una visión ascendente, incluyente y principalmente, con un irrestricto respeto a la condición humana.

\section{Consideraciones finales}

- Honduras, debe su desarrollo principalmente a la iniciativa de la pequeña empresa local. En el occidente de Honduras, la dinámica socioeconómica se mueve en función de la producción del café. Algunos productores han fortalecido sus capacidades técnicas, administrativas y financieras; sus iniciativas emprendedoras les ha permitido convertir fincas convencionales, hacia aquellas que integran diversas actividades productivas; relaciones familiares, 
gestión y fortalecimiento empresarial y un cuidado del entorno.

- El rubro del café genera en el occidente del país, cerca de dos millones de empleos. Es el proceso de reinversión de la producción del café en la pequeña propiedad familiar lo que hace posible capitalizar y crecer, aun y cuando están en una enorme desventaja estructural y en cuya cadena de valor, son quienes logran menos ingresos.

- La economía del café, con sus productos y subproductos, ofrece grandes oportunidades en investigación científica. Acercarse a estas experiencias, estados o realidades que vive la población del occidente del país, especialmente en la pequeña propiedad familiar, posibilita a estudiantes y docentes de la academia, la búsqueda de conocimientos y posibilidades a los múltiples problemas estructurales que enfrentan las comunidades de la región occidental de Honduras. Esas posibilidades cubren los aspectos técnicos, estructuras de acopio y comercialización, y principalmente, la dinámica social y ambiental que gira en torno a la cultura del café.

\section{Reconocimientos}

A las familias productoras de café, Cooperativas, a las Empresas Asociativas, a los Gremios de Productores, al IHCAFE, IICA, HWC, Municipalidades. A los compañeros del equipo de investigación de la Coordinación Regional de Investigación Científica; Kevin Javier Estévez, José Salomón Orellana, Jorge Evelio Miranda, Juan Manuel López, Lenin Reyes. A todas las personas quienes nos han abrigado y atendido con tanta bondad; a nuestras familias de la cual somos parte. A nuestros colegas del Centro Regional Universitario de Occidente, hombres y mujeres. A todos, muchas gracias. 


\section{Bibilografía}

- Alvarado, J. A. (1936). Tratado de Caficultura Práctica. Guatemala: Tipografía Nacional.

- Barahona, M. (2002). Evolución Histórica de la Identidad Nacional (2da. ed.). Tegucigalpa, Honduras: Guaymuras.

- Carranza, A. (2012). Historia y Cultura del Café en el Occidente de Honduras. Aromas, Montañas y Gente. Tegucigalpa, Honduras: PROMECAFE/IHCAFE/BID/FOMIN: Impresiones Industriales.

- Carranza, A. (2004). Palabras de Maíz y Barro. San Pedro Sula, Honduras: Diseños Impresos Múltiples DIM.

- Carranza Ramos, A. (2003). Cuando lo Segundo es lo Primero, 25 especies de plantas tradicionales del Occidente de Honduras. Tegucigalpa, Honduras: Fondo Editorial de la UPNFM.

- Castellanos, G., J. E. (1980). Atlas Geográfico de Honduras. Tegucigalpa, Honduras: ServiCopiax.

- Clifford, G. (1973). La Interpretación de las Culturas. Nueva York, USA: Basic Book Inc.

- Flores, F., Palacios, S. (2009). Honduras geográfica, Tegucigalpa, Honduras: Ramsés.

- Kluckhohn, C. (1973). Mirror for Man.Barcelona, España: Gedisa.

- Molina, G. (1985). Estado Liberal y Desarrollo Capitalista en Honduras. Tegucigalpa, Honduras: Editorial Universitaria.

- Montes, Maldonado, C. (1928). El Cultivo Racional del Café en Honduras. Tegucigalpa, Honduras: Tipografía Nacional.

- Newson, L. (2000). El Costo de la Conquista. Tegucigalpa, Honduras: Guaymuras. 
- Orellana, J., Landaverry, M., y Miranda, J. (2013). Las Fincas Integrales como Medios para Mejorar las Condiciones de Vida de los Pequeños Productores del Occidente de Honduras. Tegucigalpa, Honduras: Impresiones Industriales.

- Pérez, A., Pérez, H., Pérez, T. (1936). Homenaje a la Ciudad de Gracias a Dios, en el CD Aniversario de su Fundación. San Pedro Sula, Honduras: Tipografía Pérez Estrada.

- Rivas, R.D. (2004). Pueblos Indígenas y Garífunas de Honduras, (Una Caracterización). Tegucigalpa, Honduras: Guaymuras.

- Rivas, A. (2004). Estimation and Evaluation of plans as indicators of tropical soil quality form the knowledge of the peasants, Cauca Colombia. Alemania: Cuvillier.

- Rivas, R. (2000). Pueblos Indígenas y Garífunas de Honduras. Tegucigalpa, Honduras: Guaymuras.

- Rosa, R. (1940). Monografía del Municipio de Llama Santa Bárbara. Santa Bárbara, Honduras: Imprenta Rosa.

- Reyes, F. (1927). Anotaciones sobre el cultivo del Café. Honduras: Tipografía Nacional.

- Rubio, M. (1975). Historia del Puerto de Trujillo, volumen I. Honduras: Banco Central de Honduras.

- Stanley, I. (1994). Síntesis Monográfica del Municipio de Trinidad. Santa Bárbara, San Pedro Sula, Honduras: Imp/edit.

- Toledo, V. (1994). Agroecología, Bases Históricas y Teóricas. Lima, Perú: CLADES.

- Vallejo, A. R. (1893). Primer Anuario Estadístico 1889. Tegucigalpa, Honduras: Tipografía Nacional. 